\title{
Fluviatile Dynamik im Mittelwesertal während des Spätglazials und Holozäns
}

\author{
SuSANNE LIPPS *)
}

\begin{abstract}
Holocene, Bronze age, Iron age, terrace, Alluvial soil, chronology, lithostratigraphy, sand, silt, loam, flood sediment, clay mineral content, calcareous composition
\end{abstract}

Lower Saxony, Northwestern German Plain, Weser River Valley

Kurzfassung: Bei der Untersuchung der spätglazialen und holozänen Bildungen im Mittelwesertal wurde den Hochflutsedimenten sowie den sedimentären und sedentären Füllungen der vom Fluß verlassenen Altarme besondere Beachtung geschenkt. Dabei konnte eine Stratigraphie des Spätglazials und Holozäns erstellt werden, die durch einige grundlegende Ereignisse geprägt wird:

1. Im Spätglazial, spätestens in der Jüngeren Tundrenzeit, fand an der Mittelweser ein Umschwung vom "braided river" zum mäandrierenden Fluß statt, verbunden mit einer geringfügigen Tieferlegung der Talaue. Der Fluß lagerte nun Hochflutsedimente ab, die in der Regel sehr grobkörnig und geringmächtig sind. Ursache für den Umschwung dürfte die Erwärmung im Bölling oder Alleröd gewesen sein, die mit gleichmäßig über die Jahreszeiten verteilter Wasserführung des Flusses einherging.

2. Im jüngeren Atlantikum oder an der Wende Atlantikum/Subboreal kam es vermutlich zu einer noch näher zu untersuchenden Tieferlegung des Auenniveaus um etwa $3 \mathrm{~m}$ bei Stolzenau, die sich weiter flußabwärts, bei Verden, wegen des Effekts der Terrassenüberschneidung nicht mehr bemerkbar macht.

3. Seit der ausgehenden Bronzezeit scheint es im Einzugsgebiet der Weser verstärkt zu Rodungen gekommen zu sein. Von nun an bis weit ins Mittelalter hinein wurden an der Mittelweser tonige Hochflutsedimente (Auelehme) abgelagert, unterbrochen durch eine schwache Erosionsphase in der römischen Kaiserzeit.

4. Mit der Rodung der Talaue kam es an der Mittelweser zu einem krassen Umschwung im Sedimentationsgeschehen. Ab etwa 900 n. Chr. (bei Verden erst ab 1200 n. Chr.) wurde unter den veränderten Sedimentationsbedingungen schluffiger Auelehm abgelagert.

5. Im ausgehenden Mittelalter (etwa Mitte 14. Jh. bis um 1500) kam es zur Ablagerung von sandreichem Auelehm, wahrscheinlich als Folge der häufigen Starkregen und Überschwemmungen, die aus dieser Zeit überliefert sind.

*) Anschrift der Autorin: Dr. S. Lipps, Nieders. Landesamt für Bodenforschung, Stilleweg 2, D - 3000 Hannover.
6. Während der Neuzeit wurden entlang des nun weitgehend regulierten und eingedeichten Flusses erneut schluffige Auelehme abgelagert, die im flußnahen Bereich häufig kalkhaltig sind. Die Sedimentation dauert bis in die Gegenwart hinein an.

\section{[Late Glacial and Holocene River Dynamics in the Middle Weser Valley]}

Abstract: The investigations on Late Glacial and Holocene deposits in the middle Weser valley concentrated on highflood sediments as well as on sedimentary and sedentary fillings of abandoned river meanders. A stratigraphy of the Late Glacial and Holocene was developped in which some events are of fundamental importance:

1. During the Late Glacial, at latest in the younger Dryas period, change from a braided to a meandering river in connection with an insignificant deepening of the valley floor took place at the middle Weser river. Afterwards thin layers of coarsegrained high-flood sediments were deposited. This change may have been caused by climate improvements during the Late Weichselian (Bölling, Alleröd), when the flow of the river was almost constant during the whole year.

2. During the younger Atlantic or at the turn Atlantic/Subboreal an erosion phase is supposed to have happened which deepened the valley floor by the amount of about $3 \mathrm{~m}$ near Stolzenau. Further investigations are necessary. At the lower course of the Weser river, near Verden, the erosion phase cannot be traced because river terraces are overlapping in that area.

3. Probably clearings became more frequent in the surroundings of the Weser valley since the late bronze age. From then on until far into the middle Ages high-flood loam rich in clay was deposited at the middle Weser river. This phase was interrupted by a minor incision during the first centuries of the Christian era (Roman time in Germany).

4. Clearings in the valley floor of the middle Weser river caused a dramatic change in sedimentation. From about 900 A.C. (at the lower middle Weser river near Verden from about 1200 A.C.) a silty high-flood loam was deposited. 
5. During the late middle Ages (from the middle of the 14 th century until about 1500 A.C.) deposition of highflood loam rich in sand took place, which was probably due to heavy rains and floods whose frequent occurence at that time has been delivered.

6. In modern times again silty high-flood loams were deposited along the now vastly regulated and diked Weser river. In the vicinity of the river those loams are frequently calcareous. The sedimentation still takes place today.

\section{Inhalt}

\section{Einleitung}

2. Hochflutsedimente

2.1. Spätglaziale und altholozäne Hochflutsedimente

2.2. Der eisenzeitliche Auelehm (qh(1))

2.3. Der mittelalterliche Auelehm (qh(2))

2.4. Der neuzeitliche Auelehm (qh(3))

3. Rinnensedimente

4. Schriftenverzeichnis

\section{Einleitung}

Die Weser ist in der Vergangenheit ein Schlüsselgebiet für die Erforschung der jüngeren Flußgeschichte gewesen. Zahlreiche Untersuchungen, unter denen die Arbeiten von NATERMANN (1939a, b, 1941), NIETSCH (1955), LUTTIG (1960) und STRAUTZ (1962) besonders hervorzuheben sind, beschäftigten sich insbesondere mit den Auelehmen der Weser. Vor etwa 25 Jahren verlagerte sich der Schwerpunkt der Forschung in andere Flußgebiete, und an der Weser war eine Revision der älteren Untersuchungen der fluviatilen Dynamik des Spätglazials und Holozäns überfällig.

Die vorliegende Arbeit entstand am Niedersächsischen Landesamt für Bodenforschung (NLfB) im Rahmen eines von der DFG geförderten Forschungsprojekts unter der Leitung von Dr. C. HINZE und Dr. H. JORDAN (ausführliche Darstellung der Ergebnisse in LIPPS 1987). Das Untersuchungsgebiet erstreckt sich im Mittelwesertal von Stolzenau über Nienburg, Hoya und Verden bis Achim südöstlich von Bremen, wobei Teile des unteren Allertals eingeschlossen sind (vgl. Abb. 1). Ziel der Untersuchungen war es, die Spätglazial- und Holozänstratigraphie zu erhellen. Insbesondere die Kenntnis der jungholozänen Flußgeschichte und der Stratigraphie der Rinnensedimente konnten erweitert werden.

Die pollenanalytische Bearbeitung von 137 Proben übernahm dankenswerterweise Herr Dr. H. MÜLlER
(Bundesanstalt für Geowissenschaften und Rohstoffe, Hannover, vgl. Anhang in LIPPS 1987). Herr Dr. M. A. GeYh (NLfB) datierte 15 Proben mittels der 14C-Analyse. Auch ihm gilt mein Dank. Außerdem wurden zahlreiche sedimentologische und bodenchemische Untersuchungen von Mitarbeitern des NLfB und der Bundesanstalt für Geowissenschaften und Rohstoffe durchgeführt. Allen, die an den Untersuchungen beteiligt waren, sei hiermit gedankt.

Hilfreiche wissenschaftliche Diskussionen zum Thema wurden mit zahlreichen Mitarbeitern des NLfB und anderer Stellen, die hier nicht alle namentlich aufgeführt werden können, geführt. Ihnen allen gilt mein Dank. Schließlich bedanke ich mich bei all jenen, die durch ihren unermüdlichen Einsatz im Gelände zum erfolgreichen Abschluß der Arbeiten beigetragen haben.

Erläuterung der benutzten Abkürzungen:

$$
\begin{aligned}
& \mathrm{q}=\text { Quartär } \\
& \mathrm{h}=\text { Holozän } \\
& \mathrm{N}=\text { Niederterrasse } \\
& \mathrm{qh}(1) \text { zeitliche Gliederung } \\
& \mathrm{qh}(2) \text { holozäner Sedimentations- } \\
& \mathrm{qh}(3) \text { phasen, (1) älter (2) älter (3) } \\
& / \mathrm{T}=\text { Ton } \\
& / \mathrm{U}=\text { Schluff } \\
& / \mathrm{S}=\text { Sand } \\
& / \mathrm{k}=\text { kalkig } \\
& / \mathrm{kf}=\text { kalkfrei } \\
& / \mathrm{F}=\text { Mudde } \\
& / \mathrm{Hn}=\text { Niedermoortorf } \\
& / \mathrm{W}=\text { Wasser } \\
& / \mathrm{y}=\text { anthropogen }
\end{aligned}
$$

\section{Hochflutsedimente}

Das spätweichselzeitliche und holozäne Sedimentationsgeschehen an der Mittelweser variierte in $\mathrm{Ab}$ hängigkeit von lokalen Faktoren wie Flußgefälle, Talbreite, Nähe zur Mündung, Zufuhr von Material aus Nebenflüssen, Vegetationsgeschichte und historischer Entwicklung. Unter Berücksichtigung dieser Aspekte konnte mit Hilfe von 437 Handbohrungen eine detaillierte Stratigraphie insbesondere des jüngeren Holozäns entwickelt werden (vgl. Tab. 1), die sich auf zahlreiche pollenanalytische, archäologische und 14CDatierungen stützt. Danach hat die Sedimentation der heute im Mittelwesertal weit verbreiteten Auelehme im wesentlichen erst in der vorrömischen Eisenzeit begonnen und mehr oder weniger kontinuierlich bis heute angehalten. 


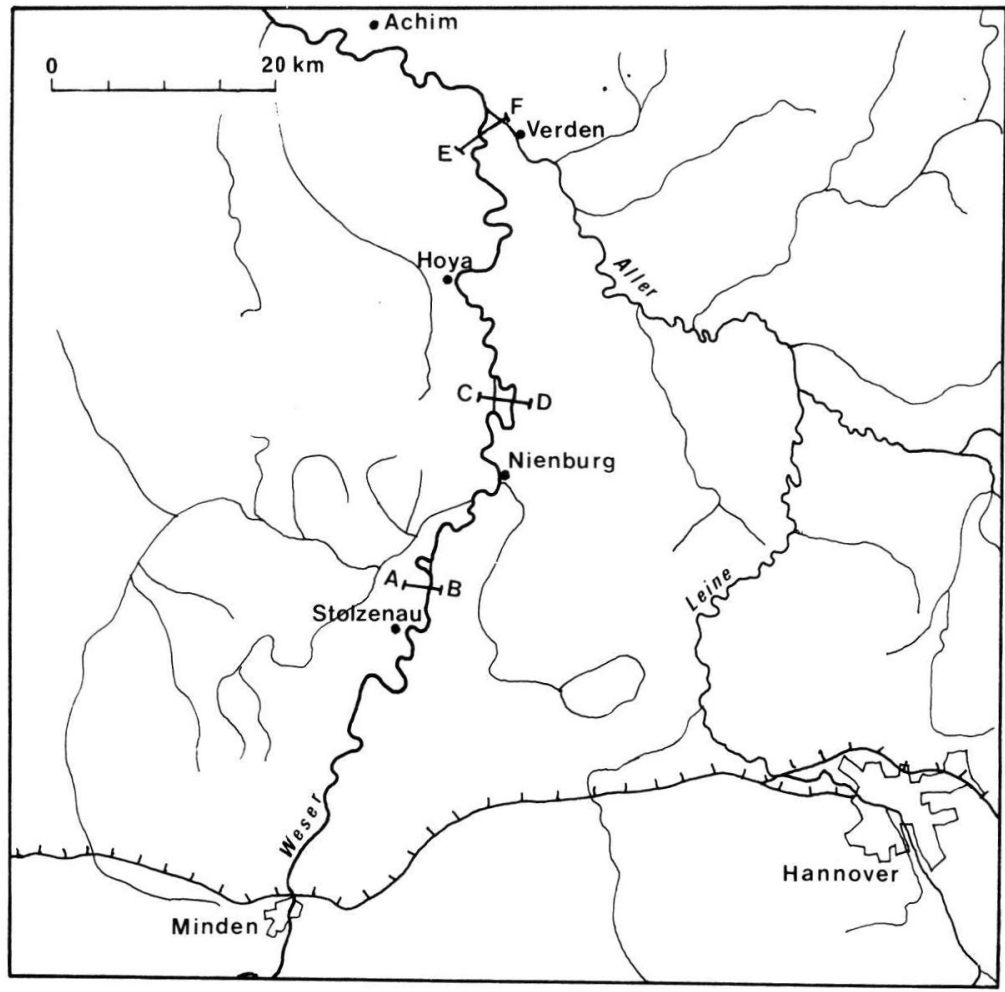

Abb. 1: Übersichtskarte.

\subsection{Spätglaziale und altholozäne Hochflutsedimente}

Im Mittelwesertal gibt es einige Hinweise auf eine spätglaziale bis altholozäne Terrasse, die bei Stolzenau größere Flächen einnimmt, wo sie nur geringfügig (etwa $1 \mathrm{~m}$ ) in die Niederterrasse eingeschnitten ist, aber um etwa $3 \mathrm{~m}$ über der rezenten Talaue liegt. Unterhalb von Hoya überschneidet sich diese Terrasse mit den jüngeren Auenterrassen (vgl. 2.2) und liegt daher ungefähr im gleichen Niveau. Dort liegen etwa $1 \mathrm{~m}$ mächtige, schwach schluffige und tonige Sande, die durch ihre deutliche Marmorierung in den verschiedensten Farben auffallen. Eine Mudde im Liegenden dieser Sedimente konnte pollenanalytisch mit einiger Wahrscheinlichkeit in die Jüngere Tundrenzeit datiert werden. Es handelt sich hier vermutlich um ein Äquivalent der häufig beschriebenen und von LANG (1973) an der Leine ins Spätglazial datierten "Hochflutlehme " bzw. der in Mitteldeutschland als „Tallehme” bezeichneten, spätweichselzeitlichen oder altholozänen Hochflutsedimente (NEUMEISTER 1964).

Es fällt auf, daß die spätglaziale bis altholozäne Terrasse von Mäandern durchzogen ist, die den jungholozänen Mäandern der Weser in Form und Ausmaß entsprechen, wogegen auf der Niederterrasse oft noch das verzweigte Rinnensystem eines "braided river" zu erkennen ist, Mäander dagegen nur ansatzweise vorhanden ist. Auf dieses Phänomen und die Folgerungen, die sich daraus ergeben, wird in Kap. 3 eingegangen.

\subsection{Der eisenzeitliche Auelehm (qh (1))}

In der vorrömischen Eisenzeit, vielleicht schon in der ausgehenden Bronzezeit, setzt im gesamten Mittelwesertal eine tonige Auelehmsedimentation ein. Die Flußaue dürfte damals noch vollständig bewaldet gewesen sein, zunehmende Rodungen im Einzugsgebiet der Weser sorgten aber durch Bodenerosion für eine verstärkte Sedimentfracht des Flusses. Dank der Bewaldung dürfte die Fließgeschwindigkeit der sich in der Aue ausbreitenden Hochwässer gering gewesen sein, weshalb es vorwiegend zu toniger Sedimentation kam.

Der Tongehalt des qh(1) beträgt im Mittel $40 \%$, vereinzelt aber auch deutlich darüber. Die Färbung des tonigen Auelehms ist rötlich, sofern in ihm keine reduzierenden Verhältnisse herrschen. Bei Stolzenau erreicht der eisenzeitliche Auelehm Mächtigkeiten von $1,5-2 \mathrm{~m}$ (vgl. Abb. 2) und liegt andeutungsweise auf einer Terrasse. Seine Ablagerung war, wie aus archäologischen Funden hervorgeht, gegen 300 
Tab. 1: Chronologie der Auelehmsedimentation

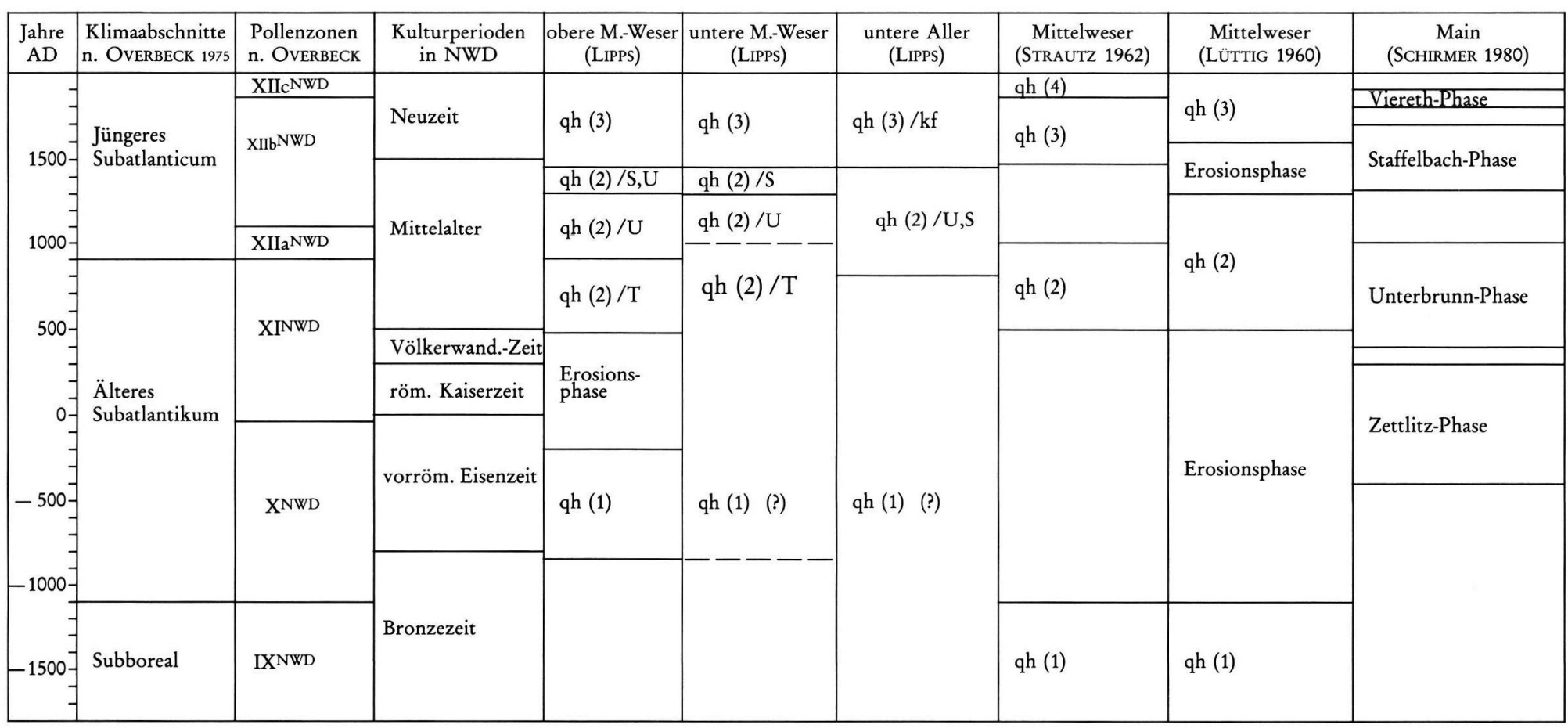

NWD $=$ Nordwestdeutschland 

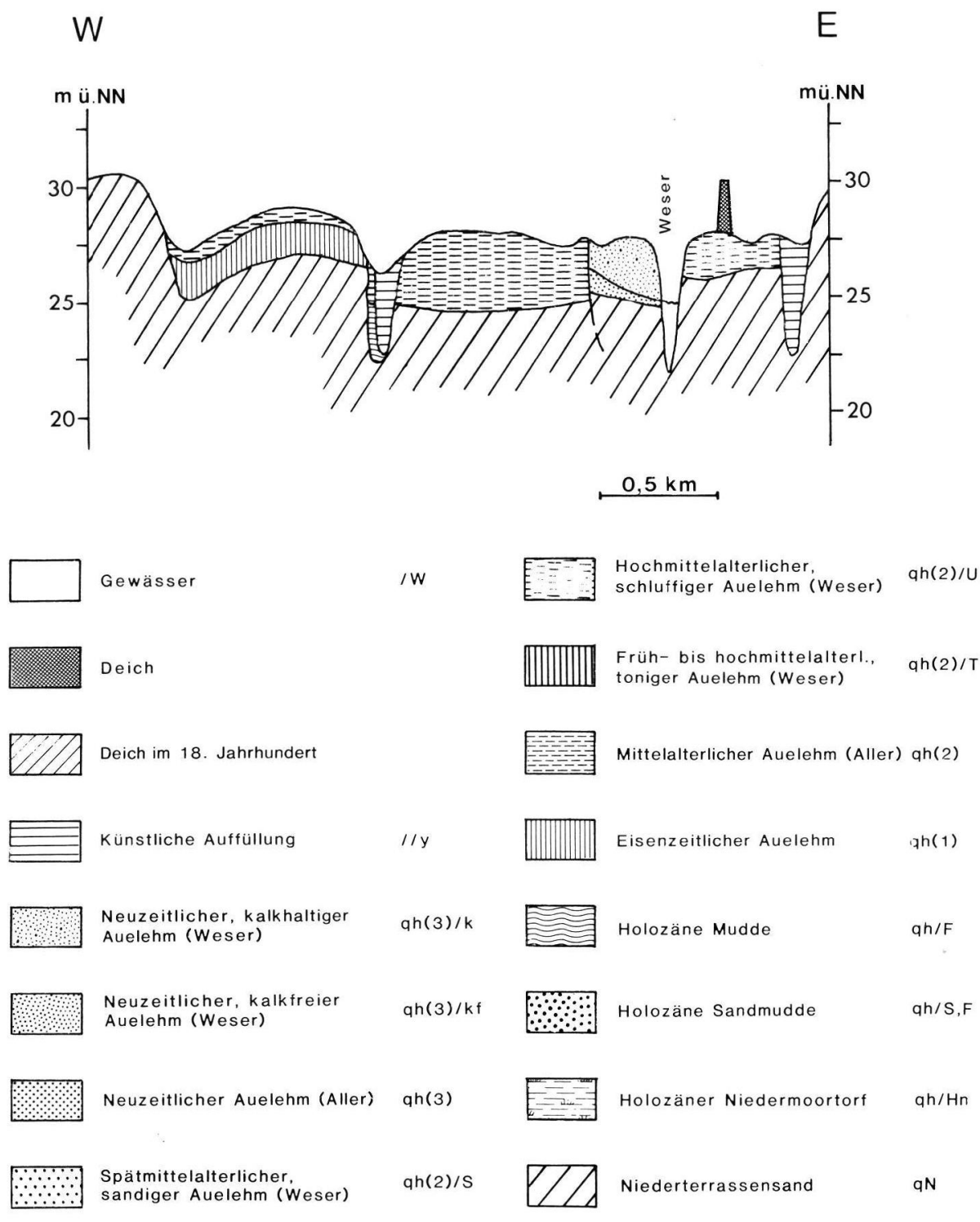

Abb. 2: Profil Landesbergen (A-B).

v. Chr. weitgehend beendet (siehe auch STRAUTZ 1962). Danach scheint es zu einer geringfügigen Tieferlegung des Auenniveaus gekommen zu sein, die allerdings von Nienburg an flußabwärts nicht mehr nachzuweisen ist (vgl. Abb. 3). Dort existiert der qh(1) als eigenständiger Auelehmkörper nicht. Es gibt lediglich vereinzelte Hinweise darauf, daß er sich an der Basis jüngerer Auelehmdecken befindet. Offensichtlich handelt es sich hier um einen Fall von Terrassenüberschneidung.

Im Allertal konnten bisher nur geringfügige Reste einer qh(1)-Sedimentation nachgewiesen werden.

\subsection{Der mittelalterliche Auelehm (qh(2))}

Im frühen Mittelalter fand im Mittelwesertal erneut tonige Auelehmsedimentation statt (qh(2)/ T). Petrographisch ist der $\mathrm{qh}(2) / \mathrm{T}$ nicht vom $\mathrm{qh}(1)$ zu unterscheiden. Im Stolzenauer Raum trennt jedoch eindeutig eine Terrassenstufe die beiden Auelehmkörper. Der qh(2)/ T wird sehr mächtig (bis über $3 \mathrm{~m}$ ) und ist insbesondere im Weser / Aller-Urstromtal bei Verden weit verbreitet (vgl. Abb. 4). Hier bilden qh(1) und $\mathrm{qh}(2) / \mathrm{T}$ vermutlich einen gemeinsamen, tonigen Auelehmkörper. Die Sedimentation scheint mehr oder weniger kontinuierlich von der späten Bronze- 


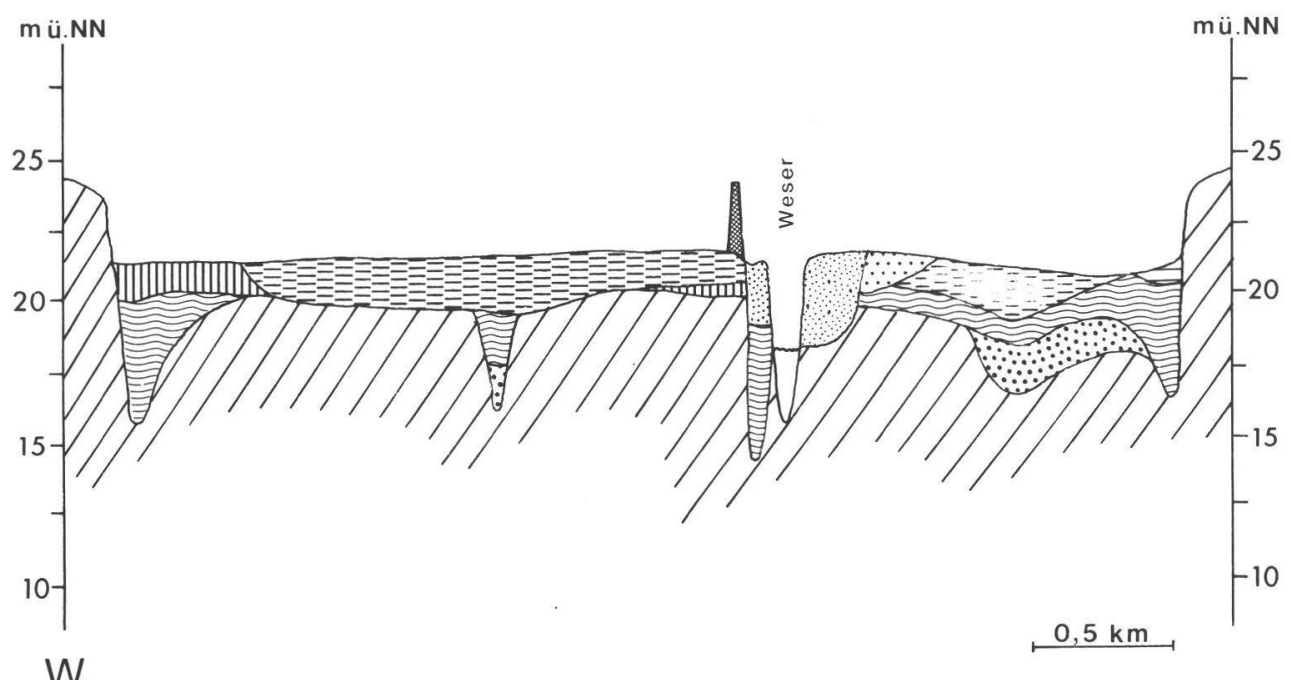

Abb. 4: (unten) Profil Klein-Hutbergen (E-F)

Abb. 3: (links) Profil Rohrsen (C-D)

\section{mü.NN}

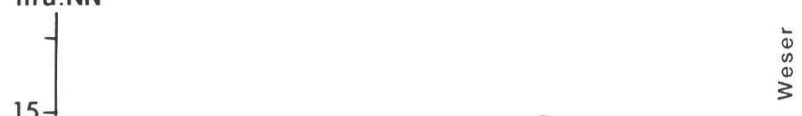

15

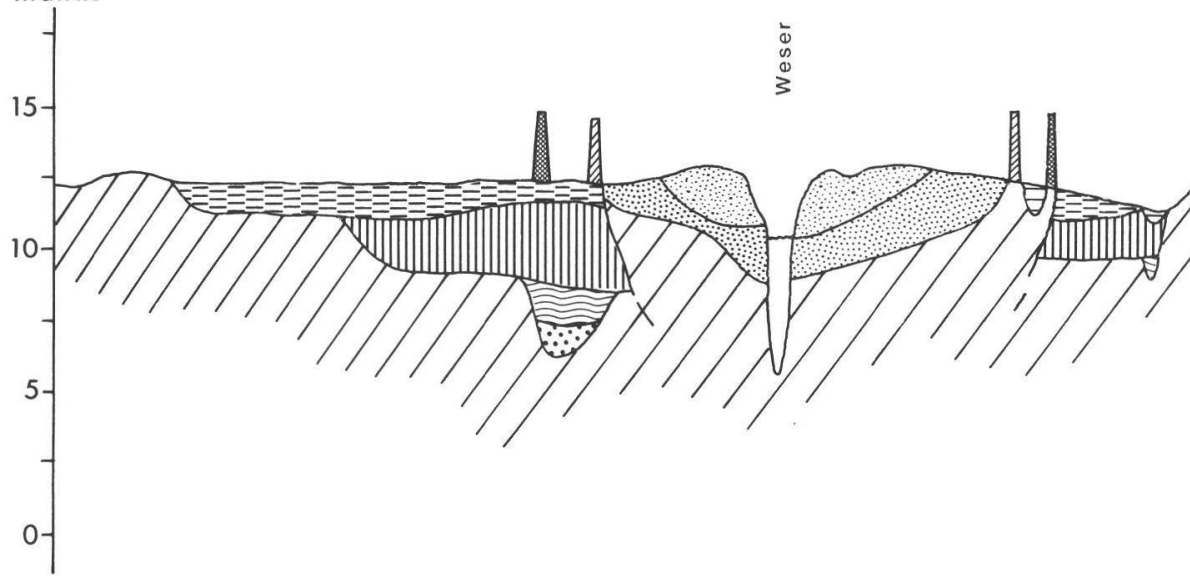


zeit bzw. frühen Eisenzeit bis ins Mittelalter hinein stattgefunden zu haben.

Die Ablagerung des $\mathrm{qh}(2) / \mathrm{T}$ dauerte bei Stolzenau etwa bis zum Jahr $900 \mathrm{n}$. Chr. an, weiter weserabwärts aber deutlich länger, bei Verden wahrscheinlich bis 1200 n. Chr. Danach kam es zu einem recht plötzlichen Umschwung im Sedimentationsgeschehen. Von nun an wurden bräunliche Auelehme mit wesentlich geringeren Tongehalten (um $25 \%$ ) abgelagert, vorherrschende Korngröße ist der Schluff $(\mathrm{qh}(2) / \mathrm{U})$. Wie konnte es zu diesem krassen Umschwung kommen? Als Erklärung bietet sich die verstärkte Rodung sowohl der Talaue als auch des Einzugsgebiets der Weser zwischen dem 9. und 12. Jahrhundert an (vgl. NATERMANN 1941; MensChING 1951). Bei Stolzenau dürfte sich schon vor dem Jahre $1000 \mathrm{n}$. Chr. die innerhalb eines oder weniger Jahrzehnte ablaufende Umstellung der Wirtschaftsweise, die von Kirche und fränkischer Grundherrschaft erzwungen wurde (vgl. NATERMANN 1941; TÜXEN 1983), auf die Auelehmablagerung ausgewirkt haben. In den gerodeten Talauen war die Fließgeschwindigkeit der Hochwässer plötzlich viel größer geworden, womit die Voraussetzungen für grobkörnigere Sedimentation geschaffen waren. Stärkere Hochwasserspitzen, verursacht durch den Fortfall der Rückhaltefunktion der natürlichen Vegetation im Einzugsgebiet, führten sogar noch zu einer 0,5 bis $1 \mathrm{~m}$ mächtigen Anlandung von qh(2)/ $U$ auf den früher weitgehend hochwasserfrei gewesenen qh(1)-Terrassen. Weiter flußabwärts scheint die Rodung der Talaue erst später stattgefunden zu haben. Auch macht sich der Rückstau der Tide, die bis Bremen reicht, wohl schon in verringerter Fließgeschwindigkeit der Hochwässer bemerkbar, ein Effekt, der für Springfluten sogar historisch überliefert ist (v. HORN 1964).

Im ausgehenden Mittelalter geht dann im gesamten Mittelwesertal die schluffige in eine schluffig-feinsandige Auelehmsedimentation (qh(2)/S) mit Sandgehalten um $30 \%$ über. Die Tongehalte liegen nur noch bei $12 \%$. Der qh(2)/S nimmt bei Stolzenau nur geringe Flächen ein, ist aber weiter flußabwärts, insbesondere im Raum Hoya, weit verbreitet. Er ist in seinem flächenhaften Vorkommen schon deutlich an den heutigen Flußlauf angelehnt und bildet darüber hinaus häufig die Füllung von tiefen Erosionsrinnen in älteren Auenbereichen. Häufig wird der qh(2)/S zur heutigen Weser hin durch die alte Deichlinie begrenzt, wie aus einer Auswertung der Kurhannoverschen Landesaufnahme aus dem 18. Jahrhundert hervorgeht. Da seit etwa $1500 \mathrm{n}$. Chr. ein geschlossener Deichschutz besteht (v. HORN 1964), der qh(2)/S aber nach pollenanalytischen Untersuchungen nicht älter als das ausgehende 14. Jahrhundert ist, kann seine Bildungszeit sehr eng eingegrenzt werden. Als
Ursache für die grobkörnige Sedimentation im ausgehenden Mittelalter kommt die Klimaverschlechterung im 14. Jahrhundert in Frage, die nach BORK (1985) die folgenreichste hygrische Klimaschwankung der letzten 13 Jahrhunderte gewesen sein soll. Außerordentliche Starkregen und damit verbundene Hochwässer, vielleicht auch die zusätzliche Rodung, die im Hochmittelalter ihren Höhepunkt erreicht hatte, könnten die Ablagerung des grobkörnigen qh(2)/S verursacht haben.

Im Allertal sind während des Mittelalters vorwiegend stark sandige Auelehme oder Auensande abgelagert worden. Eine Differenzierung in verschiedenaltrige Auelehmdecken wie an der Mittelweser ist nicht möglich. Bei den geringmächtigen tonigen Lagen, die gelegentlich dem qh(2) der Aller eingeschaltet sind, dürfte es sich um eine unter Waldbedeckung in sumpfigeren Auenbereichen entstandene Fazies handeln.

Während des Mittelalters mündete die Aller erst bei Achim in die Weser. Dies läßt sich sowohl anhand von Altarmen als auch von Allersedimenten weit unterhalb der heutigen Mündung nachweisen. Eine Vermischung des qh(2) der Weser und der Aller hat vor der damaligen Mündung nicht stattgefunden, obwohl über mehrere Kilometer hinweg eine gemeinsame Talaue benutzt wurde. Vielmehr ist die Grenze zwischen Weser- und Allerauelehmen recht scharf und wird durch zahlreiche Niederterrasseninseln markiert.

\subsection{Der neuzeitliche Auelehm (qh(3))}

Während der Neuzeit wurde im Mittelwesertal wiederum schluffiger Auelehm (qh(3)) abgelagert. Man findet ihn in einem schmalen Streifen entlang des heutigen Flußlaufs. Nur in Flußschlingen, wo durch Wanderung der Mäander in der Neuzeit noch Ausräumung älterer Auelehmdecken stattgefunden hat, nimmt er größere Flächen ein. In Flußnähe ist der qh(3) meist kalkhaltig (bis zu 5\% Kalkgehalt, $\mathrm{qh}(3) / \mathrm{k}$ ), in flußferneren Bereichen dagegen in der Regel völlig entkalkt (qh(3)/kf). Die Frischsedimentzufuhr ist dort zu gering, um den Entkalkungsprozeß aufzuhalten (vgl. OELKERS 1970).

Der $\mathrm{qh}(3) / \mathrm{kf}$ ist dem $\mathrm{qh}(2) / \mathrm{U}$ sehr ähnlich, weist aber oft einen höheren Sandgehalt $(10-20 \%)$ auf. Damit ist er immer noch deutlich feinkörniger als der $\mathrm{qh}(2)$ / S, was ein weiterer Hinweis auf klimatische Ursachen für die Grobkörnigkeit des spätmittelalterlichen $\mathrm{qh}(2) / \mathrm{S}$ ist. Bei Stolzenau scheint der qh(3) ab 1600 entstanden zu sein (vgl. STRAUTZ 1962). Von Hoya an flußabwärts ist er eindeutig an den nicht eingedeichten Bereich in Flußnähe gebunden. Da die Deiche etwa seit 1500 (s. o.) in ihrer heutigen Form 
bestehen, abgesehen von Deichbegradigungen, die nach dem 2. Weltkrieg durchgeführt wurden, muß der qh(3) in der Neuzeit entstanden sein. Seine Ablagerung dauert bis heute an, wie noch im Winter und Frühjahr 1987 nach Hochwässern zu beobachten war.

Die Aller lagerte während der Neuzeit einen stark sandigen Auelehm bis Auesand ab, in dessen Sandfraktion der Grobsand vorherrscht. Der qh(3) der Aller ist generell kalkfrei, was wohl auf seine Grobkörnigkeit zurückzuführen ist, denn im Liegenden des $\mathrm{qh}(3)$ Auelehms wurden kalkhaltig, feinklastische Rinnensedimente gefunden. Eine Vermischung von Weserund Allersedimenten findet erst einige Kilometer abwärts von der Mündung statt.

\section{Rinnensedimente}

Der mäandrierende Fluß hat während des Spätglazials und Holozäns immer wieder sein Flußbett verlagert. Die dabei entstandenen Altarme sind nach und nach verlandet. In diesen Rinnen wurden in der Regel Mudden sedimentiert, manchmal entwickelte sich ein verlandender Altarm auch zum Niedermoor. Häufig finden sich Mudden und Torfe in Wechsellagerung.

Während oberhalb von Hoya, also bevor die Weser in das Urstromtal der Aller eintritt, meist nur schmale Rinnen auftreten, die in ihren Ausmaßen dem heutigen Flußbett entsprechen, scheint das Gebiet um die Allermündung herum vor der Ablagerung der Auelehmdecken, also vielleicht bis in die Bronzezeit hinein, weitflächig versumpft gewesen zu sein.

Bei Stolzenau können zwei Generationen von Rinnen unterschieden werden (vgl. 2.1). Die Rinnenfüllungen der Mäander auf der $3 \mathrm{~m}$-Terrasse bei Stolzenau sind zwischen Spätglazial und Atlantikum vom Fluß verlassen worden. Der Fluß hat also schon unter spätglazialen Bedingungen mäandriert, was auch im Main (SCHIRMER 1980) und im polnischen Tiefland (KOZARSKI \& ROTNICKI 1977) der Fall war. Der genaue Zeitpunkt für den Umschwung vom "braided river" zum mäandrierenden Fluß läßt sich für die Mittelweser noch nicht angeben. Er liegt aber vor der Jüngeren Tundrenzeit. Im Bölling oder Alleröd könnte es also erste Mäander gegeben haben.

Die Rinnen in der rezenten Talaue sind dagegen nicht vor dem jüngeren Atlantikum, wahrscheinlich aber erst vom Subboreal an vom Fluß verlassen worden. Vermutlich kam es im jüngeren Atlantikum oder an der Wende Atlantikum/Subboreal bei Stolzenau zu einer Tieferlegung des Auenniveaus um etwa $3 \mathrm{~m}$, während die Aue ihre Höhenlage davor vom Spätglazial an und danach bis heute jeweils praktisch nicht verändert hat. Genauere Untersuchungen zu diesem Phänomen stehen noch aus. Ähnliche Beobachtungen werden auch aus anderen Flußgebieten beschrieben, so von der Ems (ROESCHMANN 1960; MÜLLER 1956), aus Mitteldeutschland (NEUMEISTER 1964) und aus dem polnischen Tiefland (KOZARSKI \& ROTNICKI 1977; KOZARSKI 1983).

Unterhalb von Hoya existiert keine morphologische spätglaziale oder holozäne Terrasse. Eine Einschneidungsphase im Holozän hat es in diesem Bereich sicherlich nicht gegeben. Jedoch sind auch hier spätweichselzeitliche Mäander dokumentiert (vgl. 2.1), allerdings im Niveau der jüngeren Rinnen. Das untere Mittelwesertal und das untere Allertal sind vor $\mathrm{Ab}$ lagerung der Auelehmdecken streckenweise flächenhaft versumpft gewesen. Die sumpfigen Streifen sind oft mehr als $1 \mathrm{~km}$ breit, also über zehnmal so breit wie das rezente Flußbett. Ein breiter Niedermoorstreifen am östlichen Talrand hat vom Atlantikum (evtl. schon vom Boreal) bis heute im Auenniveau kontinuierlich bestanden. Mit dem ausgehenden Atlantikum oder frühen Subboreal dehnte sich die Versumpfung mit Mudde- und Torfbildung dann über größere Flächen aus. Ihren Höchststand erreichte sie zu Beginn des jüngeren Subatlantikums, etwa zwischen 900 und 1200 n. Chr. Aus dieser Zeit stammen die im Mündungsdreieck von Weser und Aller weit verbreiteten, nur $0,5 \mathrm{~m}$ mächtigen Mudden, die später von Auelehm überdeckt wurden. Ab etwa 1200 beschränkte sich die Versumpfung dann wieder auf einen schmalen Streifen am östlichen Talrand.

\section{Schriftenverzeichnis}

BORK, H.-R. (1985): Mittelalterliche und neuzeitliche lineare Bodenerosion in Südniedersachsen. - Hercynia N. F., 22, 3: 259-279; Leipzig.

HORN, W. VON (1964): Deichgeschichte des Weser-AllerDreiecks. - 193 S.; Verden (Stedorfer und Westener Deichverband.

KozarsKi, S. (1983): The Holocene Generation of Paleomeanders in the Warta River Valley, Great Polish Lowlands. - Geol. Jb. A 71: 109-118, 5 Abb.; Hannover.

- \& RotNICKI, K. (1977): Valley floors and changes of river channel patterns in the North Polish Plain during the late Wurm and Holocene. - Quaestiones Geographicae, 4: 51-95; Poznàn.

LANG, H. D. (1973): Erläuterungen zur Geol. Karte von Niedersachsen 1:25000, Blatt 3323 Schwarmstedt. 78 S., 12 Abb., 13 Tab., 1 Kt.; Hannover (Nieders. L. -Amt Bodenforsch.).

LIPPS, S. (1987): Fluviatile Dynamik nordwestdeutscher Flüsse im Jungquartär. - Ber. Arch. NLfB 101 213: 66 S., 5 Abb., 4 Tab., 37 Profile, 2 Kt., Anhang von H. MÜlleR (15 S., 1 Tab.); Hannover. - [Unveröff.]. 
LÜTTIG, G. (1960): Zur Gliederung des Auelehms im Flußgebiet der Weser. - Eiszeitalter und Gegenwart, 11: 39-50, $4 \mathrm{Abb}$.; Öhringen.

MEnsChING, H. (1951): Die Entstehung der Auelehmdekken in Nordwestdeutschland. - Proc. 3 rd intern. Congr. Sediment. 5.—12. Juli 1951: 193-210, 7 Abb.; Groningen-Wageningen.

MÚlLER, H. (1956): Ein Beitrag zur holozänen Emstalentwicklung zwischen Meppen und Dörpen auf Grund von pollenanalytischen Untersuchungen. - Geol. Jb., 71: 491-504, 6 Abb., 1 Tab.; Hannover.

NatermanN, E. (1939a): Zur Geologie der Wesermarsch oberhalb Achim. - Abh. Naturw. Ver. Bremen, XXXI, 1: 154-167, 7 Abb.; Bremen.

- (1939b): Zeitbestimmung einer Flußverlagerung auf Grund der Auelehmbildung. - Abh. Naturw. Ver. Bremen, XXXI, H. 2, 326-334, 3 Abb.; Bremen.

- (1941): Das Sinken der Wasserstände der Weser und ihr Zusammenhang mit der Auelehmbildung des Wesertales. - Archiv f. Landes- und Volkskunde von Niedersachs., 9: 288-309, 8 Abb.; Göttingen.

Neumeister, H. (1964): Beiträge zum Auelehmproblem des Pleiße- und Elstergebietes. - Wissensch. Veröff. dt. Inst. Länderkunde, N. F., 21/22: 65-131, 4 Abb., 1 Tab., 8 Fotos; Leipzig.

NieTsCH, H. (1955): Hochwasser, Auenlehm und vorgeschichtliche Siedlung. Ein Beitrag auf der Grundlage des Wesergebietes. - Erdkunde, 9, 1/4: 20-39, 8 Abb.; Bonn.
OelKers, K.-H. (1970): Die Böden des Leinetales, ihre Eigenschaften, Verbreitung, Entstehung und Gliederung, ein Beispiel für die Talböden im Mittelgebirge und dessen Vorland. - Beih. Geol. Jb., 99, Bodenkdl. Beitr.: 71-151, 16 Abb., 5 Tab., 5 Taf.; Hannover.

Preuss, H. (1979): Die holozäne Entwicklung der Nordseeküste im Gebiet der östlichen Wesermarsch. - Geol. Jb., A 53: 3-84, 25 Abb., 5 Tab.; Hannover.

Roeschmann, G. (1960): Die Grundwasserböden des Emstales zwischen Rheine und Papenburg und ihre Beziehungen zur Geschichte des Emslaufes. - Geol. Jb., 77: 741-820, 2 Taf., 11 Abb., 6 Tab.; Hannover.

SCHIRMER, W. (1980): Exkursionsführer zum Symposium Franken. Holozäne Talentwicklung - Methoden und Ergebnisse. - 210 S.; Düsseldorf. - [Unveröff.]

StraUTZ, W. (1962): Auclehmbildung und -gliederung im Weser- und Leinetal mit vergleichenden Zeitbestimmungen aus dem Flußgebiet der Elbe. - Beitr. Landespflege, 1: 273-314; Stuttgart.

STREIF, H. (1975): Versuch einer Bilanzierung der Sedimentation im Küstenholozän Ostfrieslands. - Geol. Jb. A 28: 3-14, 1 Abb., 2 Tab.; Hannover.

TÜxEN, J. (1983): Vor- und Frühgeschichte / Dorfentwicklung. - In: Gemeinde Leese (Hrsg.): 800 Jahre Gemeinde Leese, 13-108; Stadthagen (Selbstverlag).

Manuskript eingegangen am 20. 8. 1987. 